\title{
Infecciones bacterianas neonatales
}

\author{
María Angélica Oto L., ; Margarita Samamé M.; \\ Rossana Camponovo C. ${ }^{3}$; María Eugenia Pinto C.3; Orquídea Arredondo B. ${ }^{3}$; \\ María Teresa Henríquez $\mathrm{H}^{3} ;$; Carmen Franco $\mathrm{S}^{4}$
}

\section{Major neonatal bacterial infections}

A prospective survey of major bacterial infections, detined as seplicemia, preumonio, uxinoly tract infections, meningilis, necrotizing enterocolitis, peritonitis, osteoartisitis and acule diarrhea, was done along o lhiee years period in a neonatal intensive cale unit of a genelal, university associaled, melropolitan hospital at Sanliago, Chile. Major bacterial infections were identified in 5.4 per I 000 live newborn infants and were significantly higher in very low birth weight intonts $144 / 1000$. Seventy three per cent of the bobies were preterm and $30 \%$ small for gestational age. Thisty one per cent of the infections were congenital and $69 \%$ nosocomial. Seplicemia was the most frequent among all olher forms of severe bacterial infeclions in this serie. Group 5 hemalyticus Streplococcus and Staphylococcus aureus were the most commonly identified infecting organisms, in congenital and nosocomial intections, respectively. Case falality raie was $31 \%$ in this serie, and autopsy was pertormed in $95 \%$ 137 of $39 ;$ falal coses. Incidence and case falality rates were signilicantly less than in a previous evaluation from yeat 1984.

(Key words: intants, newborn, preterm, infections, bacletial, septicemia.)

Las infccciones bacterianas continúan siendo un problema grave en los servicios de recién nacidos con unidad de cuidados intensivos neonatales. La complejidad dcl manejo aclual, y la disminución de la mortalidad neonatal especialmente en pacientes de muy bajo peso de nacimiento, trae consigo un aumento de las infecciones bacterianas intrahospitalarias en niños en que éste es inferior a $1500 \mathrm{~g}$. La incidencia de las infecciones bacterianas es mayor a menor peso de nacimiento, debido a que cs más intensa la deficiencia del sistema inmunitario humoral y celular'.

EI objetivo de este estudio fue determinar la incidencia, localización, etiología y letalidad de las infccciones bacterianas neonatales graves.

1. Departamento de Pediatría, Facultad de Medicina, Campus Occidente, Liniversidad de Chile. Unidad de Neonalología, Servicio de Pediatría, Hospital San Juan de Dios. SSMOc.

2. Becarios, Departamento de Pedialría

3. Unidad de Microbiologia, Facultad de Medicina, Campus Occidente. Universidad de Chile.

4. Servicio de Anatomía Palológica, Hospital San Juan de Dios.

\section{Material y Método}

Se realizó un análisis prospectivo de los pacientes atendidos en la Unidad de Neortatología del Hospital San Juan de Dios, provenientes de la matemidad del mismo hospital dursnte 3 anos, desdc el 1 de jolio de 1989 al 30 de junio dc 1992. Los pacientes incluidos corresponden a aquellos en quienes se confimaron infecciones bacterianas mayores o graves, cualquiera que fuese su peso al nacer, edad gestacional y edad cronológica, durante su hospitalización en la unidad de neonatología.

La información se registró en un protocolo cspecialmen. te diseñado con esta finalidad. Se consideraron infecciones bacterianas mayojes, graves: septicemia, bronconeurnoría, ostcoartritis, meningitis, enterocolitis necrolica, peritonitis, infección de tracto urinario y síndrome diarreico agudo. Las infecciones bacterianas mayores y graves se clasificaron en connatales e intrahospitalarias. Se dcnominaron connatales las originadas en colonización vaginal matema o infección intraamniólica, cuyos sintomas iniciales ocurrieron antes de las 48 horas de vida. Se clasíficaron como intrahospitalarias las infecciones que se manifestaron después de transcumidas 48 horas desde el nacimiento. El diagnóstico de las infecciones se basó en los signos y síntomas, exámenes de laboratorio inespecíficos, radiología, bacteriología y escudio anatomopatológico, según su localizaciốn, de acuerdo a los critcrios que se cspocifican a continuación:

Septicomia : se confirnó el diagnóstico en basc a clínica compatible, hemograma alterado y 2 hemocultivos positivos. En cl caso de Staphylococcus coagulasa negativa se requirió aislamiento del agente antes de 72 horas desde el 
momento del cultivo? I. En cl caso de Streptococcus beta hemolítico grupo $\mathrm{B}$ (agolactiae), y Listeria monocytogenes un hemoculivo positivo confirmó el diagnóstico. En los fallecidos se confirmó por hallazgos anatomopatológicos característicos: bazo friable e infiltrado inflamatorio con leucocitos polimorfonucleares en hígado y bazo.

Bronconeumonía: se diagnosticó en los recién nacidos con signos clínicos sugerentes, imagenes radiológicas persistentes, cultivo traqueal (en niños inlubados para ventilación mecánica) y un hemoculivo, ambos positivos para el mismo microorganismo y con igual susceptibilidad a los antibióticos [2]. El diagnóstico de bronconeumonia connatal se respaldó en aspirado traqueal, obtenido antes de las 8 horas de vida e inmedjatamente después de la intubación. que mostraba polimorfonucleares y bacterias al examen directo, con cultivo positivo concordante ${ }^{4}$. En los pacientes fallecidos se consideraron signos macroscópicos de bronconeumonia los pulmones śblidos de color rojo vinoso. Los signos histologicas fueron, en infecciones por Gram positivos, neutrófilos intraglveolares y acúmulos de microorganismos, en las por Gram negativos, además, la existencia de necrosis extensa.

Osteoartritis: se sospechó por manifestaciones clíni. cas, hemograma alterado, aumento importante de la velocidad horaria de sedimentación, y sumento de la proteina $C$ reactiva y se confirmó en la punción anticular con la obtencion de líquido pusulento y cultivo positivo.

Menlngltis: el diagnóstico se confinmó por manifesta. ciones clínicas compatibles, examen citoquimico del líquido cerebro espinal alterado y cultivo positivo. En el caso de estudio anatomopalológico, cuando este mostró superficie meníngea opaca, blanco amarillenta y pus.

Enterocolitis necrotica: se identificó por distensión abdominal, retención gástrica biliosa, sangre fresca en deposiciones; signos radiológicos de asa fjja o aire inirsmural (neumatosis) o apariencia lípica en cingía o aulopsia con asas intestinales delgadas, friables, cianóticas y eventualmente perforaciones.

Peritonitis: se planteó cuando se aislaron microorganismos en el cullivo peritoneal obtenido por punción abdominal, durantc cingía u necropsia. En esta úliima se observaba serosa opaca y líquido peritoneal turbio o punlento.

Infección del tracto urinario: se confirmó por cultivo positivo obtenido mediante punción vesical suprapúbica.

Diarrea: se consideró de origen bacteriano cuando se detectó un microorganismo patógeno en el coprocultivo.

En el hemograma, las alteraciones de los neutróflos se evaluaron basándose en las variaciones normales para recién nacidos comúnmente aceptadas ${ }^{3}$. Se consider 6 Irombocitopenia un recuento de plaquetas menor a $100000 / \mathrm{mm}$ cúbicos.

Esquema terapéutico: En nuestra unidad, según criteros epiderniológicos y la susceptibilidad a los antibiótcos, la terapia antimictobiana inicial para las infecciones de etiología desconocida es -en las infecciones connatales - penicilina y gentamicina. En las intrahospitalarias se emplean cloxacilina y amikacina simultáneamente Las meningitis purulentas por agente desconocido se tratan con penicilina, un aminoglicósido y cefotaxima. Una vez obıenido el microorganismo causa], la terapia entimicrobiana se mantiene o modifica según la evolución clínica y el antibiograma correspondiente. Cuando el microorgarismo aislado es Lis teria monocyrogenes la penicilina se reemplaza por ampicilina. En los recién nacidos con septicemia a Sireprococcus beta bemolítico del grupo B o Lisleria monocyto. genes, la penicilina o ampicilina se asocia a un aminoglicósido dumante los primeros 7 dias de terapia, con el fin de obtener efecto sinérgico ${ }^{\top}$. En niños con septicenia, bronconeumonía o meningitis a Pseudomonas aeruginosa se emplea la asociación de ceftazidima y amikacina. En niños con infección grave a Staphylococcus aureus meticilina-resistente se utiliza vancomicina. La dosis y duración del tratamiento con antibióticos depende de la etiología, localizzción y especialmente del peso al nacer $r^{7 \cdot 11}$.

El estudio cstadístico de las diferencias se realizó mediante el cálculo de chi cuadrado, con corrección de Yates para n menor a 25 , a un nivel de significación de alfa $5 \%$, unilateral.

\section{Resultados}

La incidencia de las infecciones bacterianas neonatales mayores fue 5,4/1 000 nacidos vivos; siendo significativamente mayor, al comparar en conjunto los menores de $1500 \mathrm{~g}$ con todos los grupos restantes ( $p<0,001$ ) (tabla 1). En los recién nacidos de muy bajo peso de nacimiento la tasa fue $143 / 1000$, correspondiendo $80 \%$ a infecciones intrahospitalarias. La incidencia de septicemia fue 3,3/1 000 nacidos vivos. En relación a la edad gestacional, eran pretérmino (menor de 37 semanas) $73 \%$ de los recién nacidos con infecciones bacterianas mayores.

El peso al nacer promedio de los niños con infecciones bacterianas graves fue $2027 \pm 925 \mathrm{~g}$ (valores extremos; $660-4750 \mathrm{~g}$ ), mediana $1885 \mathrm{~g}$. La edad gestacional promedio fue $33,4 \pm$ 4,3 semanas (24-42), mediana 33 semanas. Según las curvas de crecimiento intrauterino de Juez y cols., $66 \%$ eran adecuados, $30 \%$ pequeños y $4 \%$ grandes para la edad gestacional. Predominó

\section{Tabla 1}

Incidencia de infecciones bacterianas neonatales segun peso al nacer. 1989 - 1992

\begin{tabular}{lrcrc}
\hline $\begin{array}{c}\text { Peso al nacer } \\
\text { (g) }\end{array}$ & $\begin{array}{c}\text { Nacidos } \\
\text { vivos }\end{array}$ & $\begin{array}{c}\text { Niños con } \\
\text { infección }\end{array}$ & Tasa* & $\mathbf{p}$ \\
\hline$<1000$ & 101 & 14 & 139 & \\
$1000-1499$ & 220 & 32 & 145 & \\
$1500-1999$ & 379 & 24 & 63,3 & $<0,001$ \\
$2000-2499$ & 1166 & 21 & 18,0 & $<0,001$ \\
2500 y más & 21360 & 35 & 1,6 & $<0,001$ \\
\hline Total & 23226 & 126 & 5,4 & \\
\hline
\end{tabular}

- Por 1000 nacidos vivos 
el sexo masculino con una tasa de 6,9 para los niños y 3,8 para las niñas $(p<0,01$ ).

Durante los tres años se pesquisaron 131 infecciones bacterianas mayores en 126 nifios. Cinco recién nacidos sufricron dos infecciones en el curso de su hospitalización, uno de ellos una infección connatal y otra intrahospitalaria. en los otros cuatro las dos infecciones fueron nosocomiales. Cuarenta y un episodios de infección eran connatales (31\%) y 90 (69\%) fueron hospitalarias.

En los 41 casos de infecciones bacterianas connatales el peso al nacer promedio era $2353 \pm$ $1000 \mathrm{~g}$ ( $680-4400 \mathrm{~g})$, mediana $2340 \mathrm{~g}$; la edad gestacional promedio era $34,2 \pm 4,9$ semanas (24-42), mediana 35 semanas, y predominó el sexo masculino con una relación de $1,56 / 1$ (ns).

Los 86 niños con infecciones intrahospitalarias pesaron al nacer, promedio, $1862 \pm 841 \mathrm{~g}$ $(660-4750 \mathrm{~g})$, mediana $1727 \mathrm{~g}$. Su edad gestacional promedio fue $33,0 \pm 3,9$ semanas $(26-41)$, mediana 33 semanas. Predominó el sexo masculino con una relación de $1,93 / 1(\mathrm{p}<0,01)$.

Los síntomas de 41 niños con infecciones connatales se iniciaron al nacer en $28(68 \%)$, entre 24 y 78 horas despuếs en 11 (27\%), y más tardíamente en 2 niños. La mediana de la edad de inicio de 90 infecciones nosocomiales fue 9 días (12 horas - 105 dias). Cinco niños presentaron signos de infecciones adquiridas después de nacer en las primeras 48 horas de vida: en dos casos se rrataba de enterocolitis necrótica, una de ellas complicada con peritonitis; una septicemia a Pseudomonas aeruginosa en un recién nacido operado de atresia esofágica con fístula traqueoesofágica; dos bronconeumonías, una a Staphylococcus aureus meticitina-resistente y la otra a Staphylococcus coagulasa negativo. Diecisiete infecciones posnatales (19\%) se detectaron después de los 28 días de vida.

Las localizaciones y microorganismos causales de las 41 infecciones connatales están en la tabla 2. El principal agente etiológico fue Streptococcus beta hemolítico grupo B constituyendo el $49 \%$ de los casos. Los veinte episodios de septicemia causados por este microorganismo se asociaron a bronconeumonía en ocho, meningitis en cuatro e infección de la vía urinaria en un caso. E. coli fue el microorganismo causal de cuatro septicemias. Listeria monocytogenes fue el agente etiológico de tres septicemias, una de ellas con bronconeumonía y meningitis. Streptoccocus pneumoniae causó una septicemia con bronconeumonia y meningitis en un niño de cuya madre se obtuvo un cultivo positivo de secresión endocervical al mismo microorganismo. Dos rccién nacidos con bronconeumonía a Streptococcus beta hemolítico grupo A tuvieron derrame pleural.

En las cinco infecciones connatales con cultivo negativo, la infección se confirmó en el estudio anatomopatológico. Los seis pacientes con infecciones connatales en que no se obtuvieron cultivos positivos fallecieron; en uno de ellos no se tomaron cultivos, debido a su gravedad, y se inició terapia antimicrobiana, y en los otros cinco casos la infección se pesquisó en el estudio anatomopatológico.

Las localizaciones de las infecciones intrahospitalarias se describen en la tabla 3 . En pro-

Tabla 2

Microorganismos causales de acuerdo al cuadro clínico de infección neonatal 1989 - 1992

\begin{tabular}{llr}
\hline Cuadro clínico & Microorganlsmo & n \\
\hline Septicemia & S. beta hemolítico grupo B & 20 \\
& E. coli & 4 \\
& L. monocytogenes & 3 \\
& S. pneumoniae & 1 \\
& Cultivo negativo & 3 \\
& Sin cultivo & 5 \\
Bronconeumonía & S. beto hemolitico grupo A & 2 \\
& Cultivo negativo & 2 \\
& Sin cultivo & 1 \\
\hline \multirow{2}{*}{ Total } & & 41 \\
\hline
\end{tabular}

Tabla 3

Localización de 90 infecciones bacterianas intrahospitalarias en 86 niños $1989-1992$

\begin{tabular}{lc}
\hline Localizaclón & n \\
\hline Septicemia & 42 \\
Djarrea aguda & 17 \\
Infección tracto urinario & 13 \\
Enterocolitis necrótica & 11 \\
$\quad$ sin peritonitis : 5 & \\
$\quad$ con peritonitis: 6 & 4 \\
Bronconeumonía & 2 \\
Meningitis & 1 \\
S. piel escaldada & $90^{*}$ \\
\hline Total &
\end{tabular}

* 4 niños con 2 infecciones intrahospitatarias 
porción de $47 \%$ correspondieron a septicemia, 19\% a síndrome diarreico agudo de etiología bacteriana, y $14 \%$ a infecciones urinarias. En las 42 septicemias intrahospitalarias se aislaron 39 microorganismos: 28 Staphylococcus aureus (19 meticilina-sensible y nueve meticilina-resistente), cuatro $P$ seudomonas aeruginosa, dos $E$, coli, dos Acinetobacter baumannii, dos Enterobacter sp. y una Klebsiella pneumoniae. En cinco de 13 infecciones de la vía urinaria se confirmaron malformaciones del rinón, la vía urinaria o ambas, como enfermedad de base.

La etiología de las 90 infecciones bacterianas intrahospitalarias se encuentra en la tabla 4. Staphylococcus aureus fue el microorganismo causal en $38 \%$ de las infecciones. De las 34 cepas de Staphylococcus aureus, 22 (65\%) correspondieron a Siaphylococcus aureus meticilinasensible (SAMS) y 12 (35\%) a meticilina-resistente (SAMR). SAMS fue cl microorganismo causal de 19 septicemias: seis asociadas con osteoartritris, dos con bronconeumonía y dos con meningitis. SAMR fue el agente etiológico de nueve seplicemias: cinco asociadas con bronconeumonía, tres con osteoartritis y una con infección de la vía urinaria. $E$. coli fuc agente etiológico en dos septicemias presuntamente de origen intestinal y en cuatro infecciones urinarias. Se identificaron 16 cepas diarreogénicas, 15 correspondieron al serotipo clásico y una a una cepa enteroinvasora. Klebsiella pneumoniae fue causante de siete infecciones urinarias, una bronconeumonía y una septicemia con ventriculitis. $P_{\text {seudomonas aeruginosa se aisló en cuatro sep- }}$ licemias, una con bronconeumonía y una con meningitis, y en un caso de diarca. Acinetobacter baumannii fue microorganismo causal de 2 septicemias, una con bronconeumonía y otra con ventriculitis; como también de una infección urinaria. Staphylococcus coagulasa negativa fue el microorganismo identificado en un paciente con ventriculitis y otro con bronconeumonía. Listeria monocytogenes sc aisló en dos casos, en uno de ellos la infección fue adquirida por cl paciente en un contacto con oura recién nacida en la cuna de procedimientos de la sala de atención inmediala. El caso índice desarrolló una septicemia a Listeria monocytogenes y de su madre se obtuvo cultivo de secresión endoccrvical positivo al mismo microorganismo. El segundo caso presento meningitis a este agente etiológico y el cultivo de mucstras de su madre resultó negativo. La Listeria monocytogenes aislada en la madre y los dos recién nacidos era del serotipo IV. Los tres cultivos con desarrollos polimicrobianos correspondieron a casos de peritonitis. De los siete niños con infecciones intrahospitalarias y cultivos negativos, seis tenían enterocolitis necrótica, dos de ellos con peritonitis, uno de los cuales fallecio y el diagnóstico se confirmó en autopsia. El séptimo paciente sufrió septicemia con peritonitis, cuyo diagnóstico tambiến se confirmó en el estudio anatomopatológico. Los dos niños con infecciones posnatales en que no se tomaron muestras para cultivos fueron casos que fallecieron debido a infecciones fulminantes: enterocolitis necrótica con peritonitis, confirmada en la autopsia, y septicemia con foco primario renal en un lactante de 3 meses con peso al nacer de $950 \mathrm{~g}$ y riñón multiquístico derecho, pesquisada en el estudio anatomopatólogico, respectivamente.

Se realizó hemograma en 112 de 131 infecciones mayores ( $86 \%$ ). Se encontró, al menos, una alteración en 86 de ellos (77\%): leucopenia en 12 , neutrofilia en 60 , neutropenia en 13 , aumento de los neutrófilos inmaduros en 54, aumento de la relación de neutrófilos inmaduros a totales en 56 , vacuolización de los neutrófilos -moderada a severa - en 12 y trombocitopenia en 22. La asociación de neutrofilia y desviación a la izquierda se encontró en $50 \%$ de los hemogramas

Tabla 4

Etiología de 90 infecciones bacterianas intrahospitalarias 1989 - 1992

\begin{tabular}{|c|c|}
\hline Agente etiológico & $\mathbf{n}$ \\
\hline $\begin{array}{l}\text { S. aurews } \\
- \text { SAMS }: 22 \\
- \text { SAMR : } 12\end{array}$ & 34 \\
\hline E. coli & 22 \\
\hline K. pnewanoniae & 9 \\
\hline$P$ aeruginosa & 5 \\
\hline A. bazmannii & 3 \\
\hline S. Goagulasa negativa & 2 \\
\hline Enterobocler sp. & 2 \\
\hline L. monocylogenes & 1 \\
\hline Polimicrobjano & 3 \\
\hline Cultivo negativo & 7 \\
\hline Sin cullivo & 2 \\
\hline Total & $90 *$ \\
\hline
\end{tabular}

- 4 niños con 2 infecciones intrahospitalarias 
alterados. En 24 niños fallecidos se alcanzó a realizar al menos un hemograma: en $67 \%$ de éstos había leucopenia, neutropenia o trombocitopenia el $67 \%$.

La tabla 5 muestra la letalidad según peso al nacer y tipo de infección. La letalidad global fue $31 \%$ y en los 78 pacientes con septicemia alcanzó a $41 \%$. En los niños nacidos con peso inferior a $1000 \mathrm{~g}$ la letalidad fue $71 \%$, en los que pesaron más de $1000 \mathrm{~g}$ ésta que fluctuó entre 23 y $29 \%$. La letalidad de los niños de peso menor a $1000 \mathrm{~g}$ fue significativamente mayor que la de los niños con peso superior $(p<0,01)$. En los nintos con infección connatal la letalidad fue $51 \%$. significativamente mayor que la de los niños con infecciones posnatales, la que fue $21 \%(\mathrm{p}<0,01)$. El estudio anatomopatológico, que se realizó en casi todos -salvo dos-de Jos 39 niños que fallecieron, confírmó la infección en 31 casos y permitio descubrirla en seis (cinco connatales y una intrahospitalaria).

\section{Comentario}

En la unidad de neonatología del hospital San Juan de Dios la incidencia de infecciones bacterianas mayores disminuyó de 6,8 en $1984^{13}$ a 5,4/1 000 nacidos vivos en el trienio 1989 . 1992 (ns), considerando que en estos últimos 3 años el promedio del peso de nacimiento y de la edad gestacional fueron menores. La incidencia de septicemia fue 3,3/1 000 nacidos vivos. En Yale, de 1979-1988 la incidencia de septicemia alcanzó 3,8/1 000 debido a una mayor sobrevida, especialmente de niños con peso inferior a $1000 \mathrm{~g}$ al nacer ${ }^{14}$.

Los menores promedios del peso de nacimiento y de la edad gestacional registrados en las infecciones intrahospitalarias probablemente se deben a la mayor sobrevida de recién nacidos de muy bajo peso, que con frecuencia presentan varias afecciones y son sometidos a muchos procedimientos invasivos múltiples y hospitalizaciones prolongadas.

La septicemia como la forma clínica más frecuente de las infecciones connatales e intrahospitalarias tambiến fue descrita en una experiencia inglesa ${ }^{15}$. El principal microorganismo causal de las infecciones connatales fue Streptococcus beta hemolítico grupo B, hallazgo similar al de otros estudios $2,4,7,16$.

\section{Table 5}

Letalidad en 126 niños con infecciones bacterianas según peso al nacer y tipo de infección

\begin{tabular}{|c|c|c|c|c|}
\hline \multirow{2}{*}{$\begin{array}{c}\text { Peso al nacer } \\
g\end{array}$} & \multirow{2}{*}{$\begin{array}{c}\text { Connatales } \\
n\end{array}$} & \multirow{2}{*}{$\begin{array}{c}\text { Intrahos- } \\
\text { pitalarias } \\
\text { n }\end{array}$} & \multicolumn{2}{|c|}{ Total } \\
\hline & & & $\mathbf{n}$ & \% \\
\hline$<1000$ & $6 / 6$ & $4 / 8$ & \multicolumn{2}{|c|}{$10 / 14(71)^{*}$} \\
\hline $1000-1499$ & $1 / 3$ & $7 / 29$ & \multicolumn{2}{|c|}{$8 / 32(25)$} \\
\hline $1500-1999$ & 67 & $1 / 17$ & \multicolumn{2}{|c|}{$7 / 24$ (29) } \\
\hline $2000 \cdot 2499$ & $3 / 6$ & $3 / 15$ & \multicolumn{2}{|c|}{ 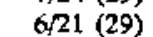 } \\
\hline 2500 y más & $5 / 19$ & $3 / 16$ & \multicolumn{2}{|c|}{$8 / 35(23)$} \\
\hline & $21 / 41$ & $18 / 85$ & \multicolumn{2}{|c|}{$39 / 126(31)$} \\
\hline
\end{tabular}

$* p<0,01$

El agente etiológico más frecuente den las infecciones hospitalarias fue el Staphylococcus aureus, siendo más de un tercio de ellos meticilina-resistente. El último caso de infección a SAMR fue en noviembre de 1990. Posteriormente, no se han pesquisado colonizaciones ni infecciones a este microorganismo, debido, entre otros factores, a la disponibilidad de insumos desechables a contar de julio de 1990 y a una buena coordinación del equipo de salud. En Estocolmo, durante 15 aftos, el principal microorganismo causal de septicemia intrahospitalaria fue también Staphylococcus aureus ${ }^{16}$.

En tres año de este estudio se detectaron pocos casos de infecciones a Staphylococcus coagulasa negativa, to que probablemente se ha debido a tres factores: criterio riguroso para la confirmación del diagnóstico, uso infrecuente de antibióticos de amplio espectro (ampicilina y cefalos. porinas de tercera generación) y excepcional de catéteres venosos centrales.

La disminución de la letalidad global fue muy importante, desde $50 \%$ en $1984^{13}$ a $31 \%$ en el trienio 1989-1992 (ns). La letalidad debido a septicemia fue $41 \%$, inferior a un estudio nacional ${ }^{17}$ y superior a dos del extranjero: $16 \%$ en el caso de Yale -en 10 años- y $33 \%$ en 15 affos en el de Estocolmo ${ }^{16}$.

Los seis casos en que las infecciones fueron descubicrtas en el examen anatomopatológico correspondieron a enfermedades fulminantes, que no permitieron estudio previo al fallecimiento. La confirmación del diagnóstico clínico en las restantes autopsias avala los criterios utilizados. 
La prevención de infeccioncs connacales, es. pecialmente a Streptococcus beta hemolítico grupo $B$, se puede realizar mediante la administración de una o más dosis de antibiótico (ampicilina o penicilina sódica por vía intravenosa) a la madre durante el trabajo de parto ${ }^{18-20} 0 \mathrm{em}$ pleando lavados vaginales con clorhexidina 1 a 6 horas antes del parto'

La incidencia de infecciones bacterianas graves en recién nacidos de muy bajo peso es muy alta y la letalidad significativamente mayor que cn los de mejor peso, lo que podría justificar el uso profiláctico de inmunoglobulina intravenosa, para disminuir la incidencia y letalidad de las infecciones intrahospitalarias 23 .

Los resultados presentados permitieron describir la incidencia, Jocalización, etiología y letalidad de las infecciones neonatales bacterianas graves en nucstra unidad, lo que es útil para conocer su realidad, compararla con otros estudios similares y establecer medidas apropiadas para disminuir la incidencia y los efectos de estas infecciones. Esto es posible si se cuenta con los recursos humanos y materiales suficientes, se trabaja en cquipo y se cumplen las técnicas de enfermería ${ }^{23}$.

\section{Resumen}

El objetivo de este estudio fue determinar la incidencia, localización, etiología y letalidad de las infecciones bacterianas neonatales graves. Se realizó un análisis prospectivo de los pacientes con infecciones confirmadas, atendidos en la Unidad de Neonatología del Hospital San Juan de Dios, provenientes de la maternidad del mismo hospital, durante 3 años. La incidencia fue 5,4/1 000 nacidos vivos, siendo significativamente mayor en menores de $1500 \mathrm{~g}$ (144/1 000). Fueron pretérmino el $73 \%$ y pequeños para la edad gestacional el $30 \%$. Las infecciones connatales correspondieron a $31 \%$ y las intrahospitalarias a $69 \%$ del total. La forma clínica más frecuente fue la de septicemia. Los agentes causales más frecuentemente identificados fueron, entre las infecciones connatales, Streptococcus beta hemolítico del grupo $\mathrm{B}$ y, en las intrahospitalarias, Staphylococcus aureus. La letalidad alcanzó $31 \%$ y se realizó estudio anatomopatologico en $95 \%$ de los fallecidos. La in- cidencia y la letalidad disminuyeron en comparación al estudio de 1984.

(Palabras clave: recién nacido, pretérmino, infecciones, septicemia.)

\section{Agradecimientos}

Al Dr. Sergio Vaisman W, por la revisión crítica de cste manuscrito.

\section{Referencias}

1. Cairo MS, Worcester C, Reuker $R$, et al: Role of circulating complement and polymorphonuclear leukocyte transfusion in treatment and outcome in critically ill nconates with sepsis. J Pediatr 1987; 110: 935-941.

2. Webber $S$, Wilkinson AR, Lindsell D, Hope $P L$, Dobson $S R M$, fsaacs $D$ : Neonatal pneumoniac. Arch Dis Child 1990; 65: 207-211.

3. Murson DP, Thompson TR, Johnson DE et al.: Coagulase-negative staphylococcal septicemia: experience in a neonalal intensive care unit. J Pediatr 1982 ; 101 ; 602 605 .

4. Sherman MP, Goetzman BW, Ahlfors $C E$, and Winnberg $R P$ : Traqueal aspiration and its clinical correlates in the diagnosis of congenital pneumoniae. Pediatrics 1980; 65: $258-263$.

5. Mamroe BL, Weinberg $A G$, Rosenfeid $C R$, at af.: The neonatal blood count in heaith and disease. I Reference values for neutrophilic cells. J Pedia1r 1979; 95: 89-98.

6. Albin AR, Kushner JH, Muphy A, Zippin C: Platelet enumeration in the neonatal period. Pediatrics 1961: 28: 822-824

7. Gandy $G$, Rennie $J$ : Antibiotic treatment of suspected neonatal meningitis. Arch Dis Child 1990; 65: 1-2.

B. Short A. Tarlow MJ Antibiotic treatment of suspected neonalal meningitis (Letlers). Arch Dis Child 1990; 65: 810 .

9. lsaacs $D$ and Wilkinson $R$ : Antibiotic use in the neontal unit. Arch Dis Child 1987; 62: 204-208.

10. Isaacs $D$, Wilkinson AR, Moxon ER: Duration of antibiolic courses for neonates. Arch Dis Child 1987; 62: 727.728.

11. Prober ChG, Stevenson DK, Benitz UF' The use of antibiotics in the neonates weighing less than 1.200 grams. Pediatr Infect Dis 1990: 9: 111-121.

12. Juez $G$, Lecero E, Ventura Junca P, Tapia JL, Gonzailez $H$, Winter $A$ : Estudio neonatal del crecimiento intrauteríno en 11.543 recién nacidos chilenos de clase media. 1978-1987. Rev Chil Pedials 1989; 60: 198-202.

13. Tellerias L, Oto MA, Lagos E, Mérudez E: Infecciones bacterianas neonstales: magnitud y aspectos clínicos. Rev Chil Pediatr 1989; 60: 262-266.

14. Gladsione IM. Ehrentronz RA, Edberg SST and Baltimore RS: A ten year review of neonatal sepsis and comparison with the previous fifty-year experience. Pediatr Infect Dis J 1990; 9: 819-825.

15. Oto A: Major bacierial infection in a referral neonatal inten sive care unit. J Infect 1982; 5: 117.126. 
16. Bennet R, Erikson $M$, Melin $B$ and Zetterström $R$ : Changes in the incidence and spectrum of neonatal septicemia during a fifteen-year period. Acta Paedialr Scand $1985 ; 74: 687-690$.

17. Enríquez N. Rizzardini $M$ : Scpticenia en upa unidad de cuidado intensivo nconatal. 1990-1991. Pediatría 1992; $35: 24-29$.

18. Yow MD, Mason EO, Leeds LW, Thompsan PK, Clark DJ. Gardner $S E$ : Ampicillin prevents intrapartum Iransmission of group B streptococcus. I A M A 1979; 241: $1245-1247$.

19. Bayer $K M$ and Gottoff $S P$. Prevention of early onset neonatal group $B$ strcptococcal diseasc with selective intrapanum chemoprophylaxis. N Engl J Med 1986; 314: $1665-1669$.

20. Commitiee on Infectious Diseases and Committee on Felus and Newborn: Guidelines for prevention of Group
B streptococcal (GBS) infection by chemoprophylaxis. Pediatrics 1992; 90: 775-778.

21. Burmar LG, Christensen $P$, Christensen $K$, Fryktund $B$, Heigesson AM, Svenningsen NW, Twlis $K$ : Prevention of excess neonatal morbidity associated with group B streptococci by vaginal clorhexidine desinfecton during labour. Lancel 1992; 340; 65-69.

22. Baker CJ, Melish ME, Holl RT, Casto DT, Vason U, Givner LB and the Multicenter Group for the Study of Immune Globulin in Neonates: Intravenous immune globulin for the prevention of nosocomial infection in low-birth-weight neonates. N Engl J Med 1992; 327 : 213-219.

23. Oto MA, Pinto ME, Marínez V y cols. Control de infecciones por Staphylococcus aureus resistentc a melicilina en una unjdad de neonatología. Rev Chil Pediatr 1992; 63: 134-138.

Esta publicación está disponible en copias de microfilms de 16 y $35 \mathrm{~mm}$ y microfichas de $105 \mathrm{~mm}$, las que pueden solicitarse a:

University Microfilms International

300 North Zeeb Road

Ann Arbor, Michigan 48106, USA.

This journal is also available in $16 \mathrm{~mm}$ microfilm, $35 \mathrm{~mm}$ microfilm and 105 mm microfilm copies through

University Microfilms International.

300 North Zeeb Road,

Ann Arbor, Michigan 48106, USA. 